\title{
Tubular J-aggregates of cyanine dyes in the near-infrared
}

Austin D. Bailey[a], Arundhati P. Deshmukh[a], Timothy L. Atallah ${ }^{[b]}$, Monica Pengshung ${ }^{[a]}$, Ulugbek Barotov ${ }^{[c]}$, Ellen M.

Sletten $^{[a]}$, and Justin R. Caram*[a]

[a] Mr. Austin Bailey, Dr. Arundhati Deshmuklh, Dr. Monica Pengshung, Dr. Ellen Sletten, Dr. Justin Caram Department of Chemistry and Biochemistry

University of California Los Angeles

Los Angeles, CA, 90024

E-mail: jcaram@chem.ucla.edu, sletten@chem.ucla.edu

[b] Dr. Timothy Atallah

Department of Chemistry and Biochemistry

Denison University

Granville $\mathrm{OH}, 43023$

[c] Mr. Ulugbek Barotov

Department of Chemistry

Massachusetts Institute of Technology

Cambridge, MA 02139

\begin{abstract}
Developing improved organic infrared emitters has wide-ranging applicability in fields such as bioimaging or energy harvesting. We synthesize redshifted analogues of C8S3, a well-known cyanine dye that self assembles into tubular aggregates which have attracted widespread attention as artificial photosynthetic complexes. Despite the elongated dye structure, the new pentamethine dyes retain their tubular self-assembly and emit at near-infrared wavelengths. Cryo-electron microscopy and detailed photophysical characterization of the new aggregates reveal similar absorption lineshapes with $\sim 100 \mathrm{~nm}$ of redshift, as well as supramolecular morphologies that resemble their trimethine counterparts; the pentamethine aggregates generally show more disorder and decreased superradiance, suggesting that more ordered structures yield more robust photophysical properties. These results provide design principles of superradiant organic emitters, expand the chemical space of near-infrared aggregates, and introduce two additional wavelength-specific antennae as model systems for study.
\end{abstract}

\section{Introduction}

Molecular aggregates are non-covalently bound self-assemblies of organic chromophores known for their strong absorption and emission properties. The relative arrangement of the dyes deeply affects the excitonic/photophysical properties, e.g. a completely cofacial arrangement of dyes leads to an $\mathrm{H}$-aggregate (less emissive species blueshifted relative to the monomer), whereas a slipped arrangement of chromophores yields $\mathrm{J}$-aggregation (highly emissive species redshifted relative to the monomer). ${ }^{1,2} \mathrm{~J}$-aggregates are of great importance due to their high oscillator strength, radiative rates, and brightness, making them well-suited for photophysical applications such as penetrative imaging or energy harvesting. ${ }^{3-6}$ Additionally, J-aggregates can take on many supramolecular morphologies such as sheets, ribbons, or tubes, leading to different photophysical properties. $^{7,8}$

In particular, tubular J-aggregates show narrow spectral features indicative of only weak disorder-characteristics also identified in nature's most efficient photosynthetic systems. ${ }^{9-13}$ Additionally, tubular aggregates have the advantage of a $2 \mathrm{D}$ density of states while maintaining directional energy flow. ${ }^{14,15}$ This work marks one of the first few examples of a cyanine dye that assembles into tubular J-aggregates (Figure 1A) with emission in the near-infrared (NIR, 700-1000 nm) ${ }^{16-18}$, a region where enhanced light penetration enables technological applications. ${ }^{19-21}$ 

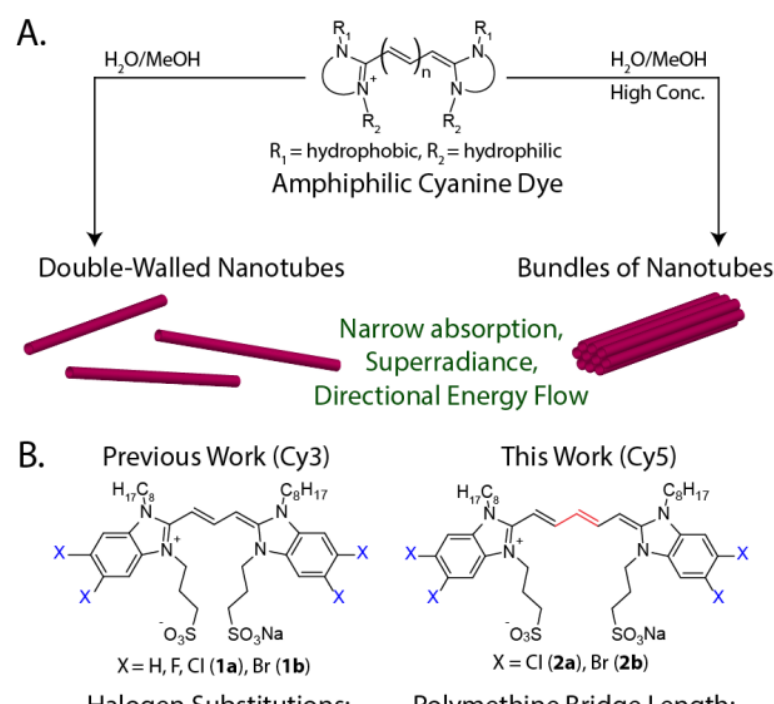

Halogen Substitutions: Polymethine Bridge Length:

Modulate Tube Diameter Redshift, Preserve Morphology

Figure 1. A. Self-assembly of amphiphilic cyanine dyes into J-aggregate nanostructures. B. Synthetic modifications on C8S3 (1a) in prior work (modifying halogen substitution/placement) and this work (trimethine to pentamethine).

To address this challenge, we modified the structure of an extant chromophore, known as C8S3 (Figure 1B, structure 1a), that forms superradiant tubular aggregates in the visible region. C8S3 was first introduced to the literature in $1997^{22}$ and has since been studied extensively as a model system for as exciton transport, antenna effects, superradiance and quantum coherence. ${ }^{14,23-30}$ Recent work used synthetic modifications on the C8S3 monomer to affect aggregation properties; larger halogen substitutions have been shown to increase the tube diameter, while placement of the halogen atoms was found to be critical in achieving $\mathrm{H}$ - vs. J-aggregation. ${ }^{31,32}$ Here, we expand this body of work by synthesizing elongated C8S3 dyes with redshifted absorption and emission.

The traditional method to bathochromically shift cyanine dyes is to increase the polymethine chain by one vinylene unit, thereby inducing a $\sim 100 \mathrm{~nm}$ redshift. ${ }^{33}$ We use this method to synthesize pentamethine dyes $\mathbf{2} \mathbf{a}$ and $\mathbf{2} \mathbf{b}$ (as opposed to trimethines $\mathbf{1 a}$ and $\mathbf{1 b}$ in the past) based on the C8S3 heterocycle, allowing us to access tubular aggregates of cyanine dyes with NIR emission. Additionally, we explore how that modification (i.e. trimethine to pentamethine) affects the self-assembly and photophysical properties of the aggregates.

\section{Results and Discussion}

The C8S3 heterocycles $(\mathbf{S 5}, \mathbf{S 1 0})$ and $\mathbf{1 a} / \mathbf{1} \mathbf{b}$ were synthesized via a literature precedent. ${ }^{31}$ The pentamethine cyanines $\mathbf{2 a}$ and $\mathbf{2 b}$ were synthesized by heating the aforementioned heterocycle (S5, S10) and electrophilic linker (malonaldehyde bisphenylimine $\mathrm{HCl}$ ) in the presence of a sterically hindered base (1,8-diazabicycloundecene, DBU) in dimethylformamide (Scheme S1). A heptamethine analog of the chlorine derivative was briefly investigated, but ultimately not pursued due to rapid degradation in methanol, which prevented aggregation and further characterization (Scheme S10, Figure S1).

In methanol, the pentamethine dye monomers absorb at $\lambda_{\max }=616 \mathrm{~nm}(\mathbf{2 a})$ and $614 \mathrm{~nm}(\mathbf{2 b})$ as opposed to the trimethine dyes with $\lambda_{\max }=522 \mathrm{~nm}(\mathbf{1 a})$ and $523 \mathrm{~nm}(\mathbf{1 b})$. Prior work on molecules 1a and $\mathbf{1 b}$ has shown that the two primary aggregate absorption features occur from the assembly of long double-walled nanotubes (DWNT) and the subsequent bundling of those nanotubes. The DWNT produces several absorption peaks, namely at 599 and $589 \mathrm{~nm}$, corresponding to the inner and outer walls of the tube respectively. ${ }^{26}$ Additionally, both the inner and outer wall show features which have been assigned to parallel $(599 \mathrm{~nm})$ and perpendicular (580 nm) excitonic transitions, which are indicative of macroscopic exciton delocalization around each tubular structure. ${ }^{34}$ The bundled nanotubes retain a prominent $603 \mathrm{~nm}$ feature but also have a less intense, broad absorption around $580 \mathrm{~nm}$.

To probe the aggregate morphologies of the new pentamethine dyes, we both screened $\mathbf{2 a}$ and $\mathbf{2 b}$ at varied concentrations while maintaining a constant methanol/water ratio (Figure 2A). ${ }^{35}$ Aggregates of each chromophore were prepared by first creating a stock solution of the dye in methanol. To a small volume of this stock solution was added ultrapure $\mathrm{H}_{2} \mathrm{O}$. The final concentration and methanol/water ratio in each solution were varied. A complete procedure for preparing aggregates is included in SI Section 4. UV-Vis absorption was used to characterize the aggregates, which display a different spectral signature for each morphology. 
In this exploration, we found that the pentamethine dye aggregates display similar photophysical properties to the trimethine dyes, forming two primary aggregates. We then employed non-negative matrix factorization (NNMF), an algorithm which allows us to estimate the relative contributions of each species in the spectra, to extract the underlying basis spectra (Figure $2 \mathrm{C}$ ) of the concentration series data in Figure $2 \mathrm{~A}$. Figure $2 \mathrm{D}$ plots the mole fraction of each isolated aggregate as a function of concentration, showing that the two distinct aggregate morphologies can be prepared at 0.01 and $1 \mathrm{mM}$. Absorption spectra for the monomers and isolated aggregates of $\mathbf{1 a}, \mathbf{1} \mathbf{b}, \mathbf{2} \mathbf{a}$, and $\mathbf{2} \mathbf{b}$ are shown in Figure 3.

The first aggregate of $2 a$ shows distinct features at 740 and $731 \mathrm{~nm}$ (achieved at low concentration, $0.01 \mathrm{mM}, 10 \% \mathrm{methanol}$ ), while the other has broad absorption around $715 \mathrm{~nm}$ and retains the $745 \mathrm{~nm}$ feature (achieved at higher than $0.1 \mathrm{mM}, 10 \%$ methanol).
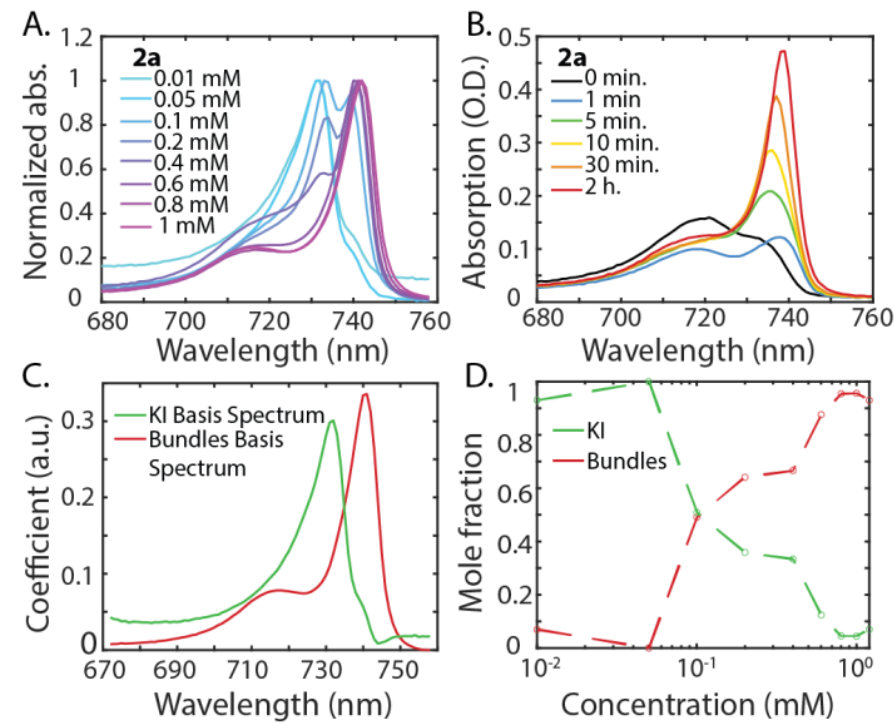

Figure 2. A. Normalized absorption of variable concentration samples of 2a. All samples created using $10 \% \mathrm{MeOH}, 90 \% \mathrm{H}$ section 4. B. Kinetic time series of a 2 a aggregate created at $10 \% \mathrm{MeOH}, 90 \% \mathrm{H}_{2} \mathrm{O}$ and $0.05 \mathrm{mM}$ dye. C. Basis spectra for kinetic intermediate and bundles of $\mathbf{2 a}$ achieved through non-negative matrix factorization of the concentration series in Figure 2A. D. Plot of the normalized relative mole fraction of kinetic intermediate vs. bundles changing as a function of concentration.

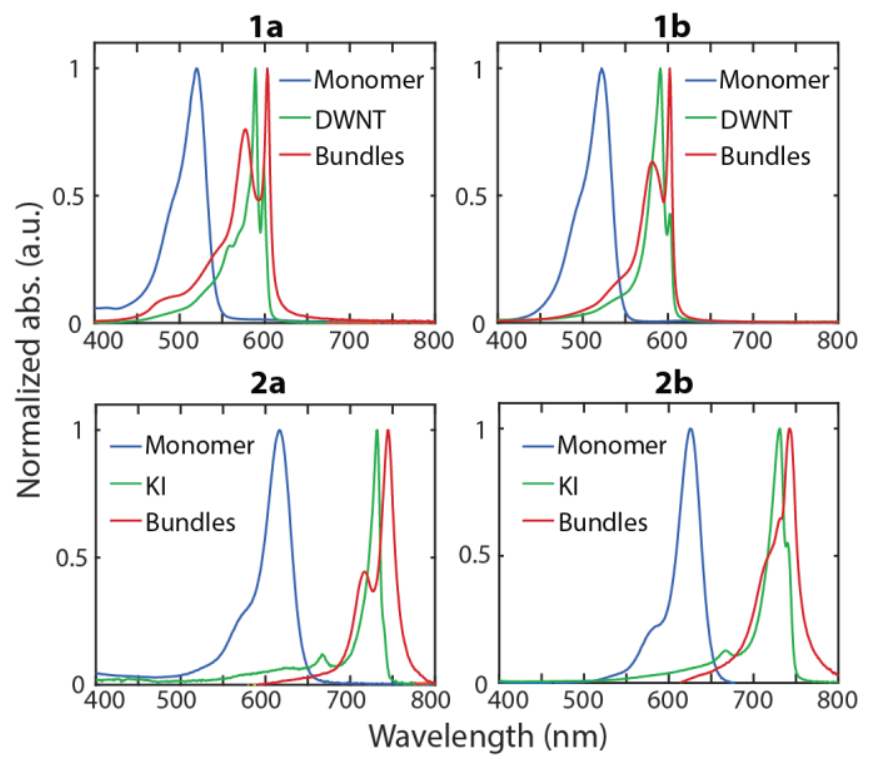

Figure 3. Morphological characterization of monomers and aggregates of $\mathbf{1 a}, \mathbf{1} \mathbf{b}, \mathbf{2} \mathbf{a}$, and $\mathbf{2 b}$. Absorption spectrum of trimethine (1a and $\mathbf{1 b}$ ) and pentamethine $(\mathbf{2 a}$ and $\mathbf{2 b})$ dye monomers $(100 \% \mathrm{MeOH})$ and aggregates (DWNT = double-walled nanotube, $\mathrm{KI}=\mathrm{kinetic}$ intermediate).

Upon identifying appropriate conditions for each aggregate with unique spectral signatures, we performed cryo-EM to determine their supramolecular morphologies. Cryo-EM for the bundled aggregate morphologies of $2 \mathbf{a} / \mathbf{2} \mathbf{b}$ and DWNT morphologies of $\mathbf{1 a / 1} \mathbf{b}$ is shown in Figure 4; details on cryo-EM technique are in SI Section 6 . We originally hypothesized that, 
like 1a, $\mathbf{2 a}$ and $\mathbf{2 b}$ would form DWNTs and bundles of nanotubes. The imaging experiments for the high concentration aggregate clearly showed bundles analogous to 1a; however, the aggregates prepared at $0.01 \mathrm{mM}$ (with features at 746 and $731 \mathrm{~nm}$ ) did not have sufficient sample density to determine morphology via EM. Additionally, at higher concentrations, these aggregates were found via UV-vis to rapidly convert to the bundled morphology (Figure 2B). We hypothesize that this shortlived kinetic intermediate (KI) represents DWNTs, though we were unable to confirm this based on electron microscopy.

Thus, increasing the length of the polymethine bridge has a strong effect on the supramolecular packing of these chromophores. Trimethines $\mathbf{1 a}$ and $\mathbf{1} \mathbf{b}$ clearly have a propensity to form individual DWNTs, while pentamethines $\mathbf{2 a}$ and $\mathbf{2} \mathbf{b}$ almost exclusively form bundles. We hypothesize that the pentamethine dyes aggregate more readily due to the increase in size (more pi-pi stacking area) and hydrophobicity, as well as increased polarizability. Additionally, the bundles are likely the thermodynamic minimum of these aggregates, and therefore stronger non-covalent interactions in the assembly may mean a lower kinetic barrier to bundle formation. Overall, we observed few differences in the aggregates formed from $\mathbf{2 a}$ and $\mathbf{2 b}$, with the exception of broader linewidths for the brominated dyes, possibly indicating a higher level of disorder in those nanostructures.

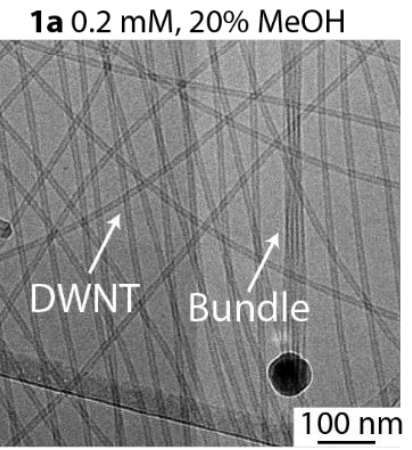

2a $1 \mathrm{mM}, 10 \% \mathrm{MeOH}$

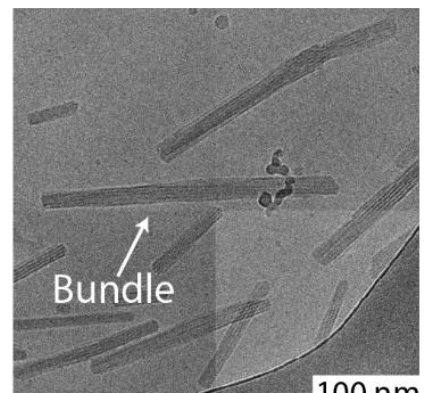

$100 \mathrm{~nm}$ 1b $2 \mathrm{mM}, 20 \% \mathrm{MeOH}$

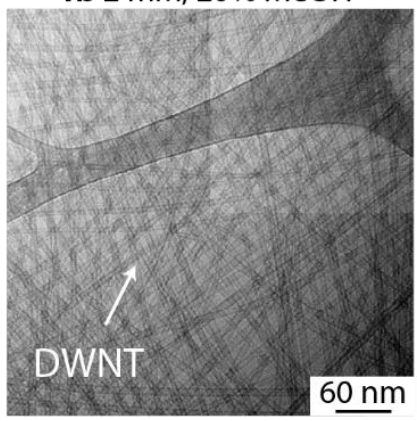

2b $0.1 \mathrm{mM}, 10 \% \mathrm{MeOH}$

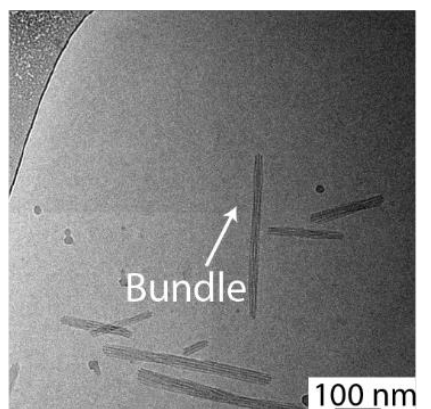

Figure 4. Cryo-electron microscopy images of trimethine double-walled nanotubes and pentamethine bundles.

Following the identification of the various aggregate morphologies, we probed the photophysical properties of $\mathbf{2 a}$ and $\mathbf{2 b}$ in comparison to 1a and $\mathbf{1 b}$ (Table 1). We used quantum yields $\left(\mathrm{QY}, \Phi_{\mathrm{F}}\right)$, energy gaps $\left(E_{g}\right)$, and fluorescence lifetimes $(\tau)$ to obtain the transition dipole moment $(\mathrm{TDM}, \mu)$ for each compound's electronic transitions, as well as each aggregate morphology's excitonic transitions. Transition dipole moments are a direct measure of how strongly these materials interact with light, making this value a critical component of this analysis. The emission spectra of $2 \mathrm{a}$ are given in Figure $5 \mathrm{~A}$, as well as the lifetimes and fittings in Figure 5B. A full explanation of the photophysical characterization and derivation of TDM can be found in SI Sections 7-9. Photophysical data used in the calculation of TDM are given in Table 1.

The transition dipole moments for all monomers and aggregates are detailed in Figure $5 \mathrm{C}$. Using these TDM values, we calculated the superradiance parameter $\eta_{S R}$ for each dye, as shown in equation 1.

$$
\eta_{S R}=\frac{\mu_{a g g}{ }^{2}}{\mu_{m o n}{ }^{2}}=\frac{k_{r, a g g} E_{g, m o n}{ }^{3}}{k_{r, m o n} E_{g, a g g}{ }^{3}}
$$

Although previous works have used different definitions for superradiance in $\mathrm{J}$-aggregate systems ${ }^{14,36,37}$, we maintain that this method offers the advantage of factoring in the difference in energy gap, which results in an intrinsic change in radiative rates due to the redshift from monomer to aggregate. ${ }^{38}$ The $\eta_{S R}$ parameter is also a measure of the coherence length for the 
fluorescent state, or the number of monomers over which the Frenkel excitons delocalize. ${ }^{39}$ This value is rigorously defined only for linear aggregates, though it is a useful metric for all aggregate morphologies.
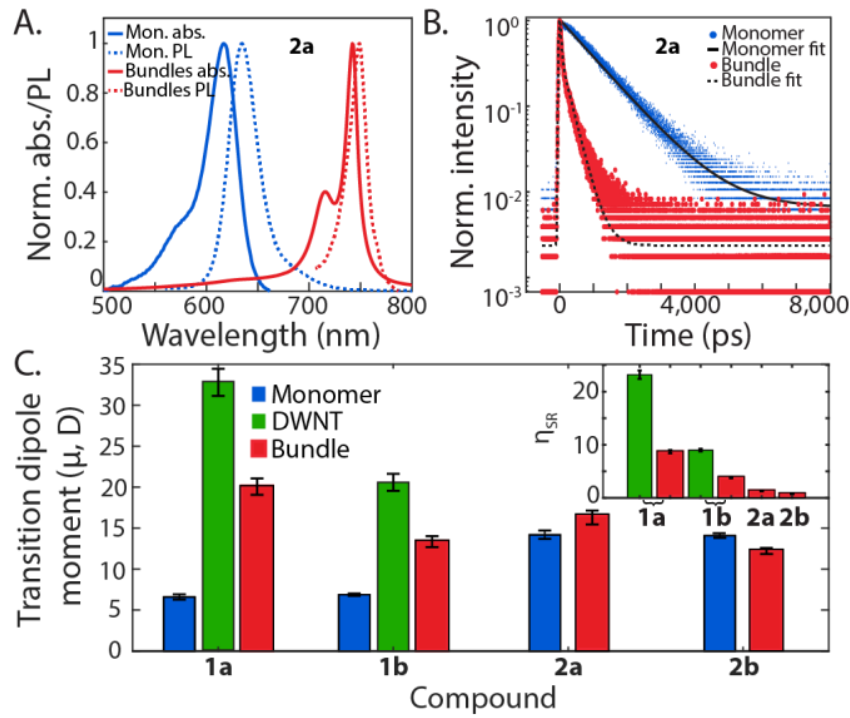

Figure 5. Photophysical characterization of monomers and aggregates of $\mathbf{2 a / 2 b}$. A. Absorption (solid) and photoluminescence (dotted) spectra for 2a monomer (methanol) and bundle (methanol/water). B. Monomer lifetime, bundle lifetime, and corresponding fits for 2a. C. Transition dipole moments for all monomers (blue) and aggregate morphologies (green, red). (Inset: superradiance parameter $\eta_{S R}$ for each aggregate morphology.)

Figure $5 \mathrm{C}$ demonstrates that the trimethine aggregates possess transition dipole moments up to 5 times larger than their monomer counterparts; this corresponds to high superradiance parameters ( 23 for 1a DWNT). However, for the pentamethine dyes, the aggregate transition dipoles are comparable to those of the monomers. This accordingly leads to $\eta_{S R}$ values that are closer to $\mathbf{1}$, meaning that they are less superradiant (or in the case of $\mathbf{2} \mathbf{b}$, subradiant). These data can be attributed to the higher disorder in aggregates of $\mathbf{2} \mathbf{a}$ and $\mathbf{2} \mathbf{b}$ which leads to decreased exciton delocalization despite retaining redshifted emission. We also observe that the brominated aggregates are less superradiant than their chlorinated counterparts; we hypothesize this is due to the additional energetic disorder, which is indicated by the broader absorption linewidths and also via structural disorder in the trimethine aggregate cryo-EM.

Table 1. Photophysical data for monomers and aggregates of dyes 1a, 1b, 2a, and $\mathbf{2 b}$. Quantum yield $\left(\Phi_{F}\right)$ error was taken as the standard deviation of triplicate measurements. Lifetime $(\tau)$ error was taken from the $95 \%$ confidence interval in fitting. Error in radiative rate $\left(k_{r}\right)$ and superradiance parameter $\left(\eta_{S R}\right)$ were propagated from the original lifetime and quantum yield measurements.

\begin{tabular}{cccccccc}
\hline Compound & Morphology & $\Phi_{F}$ & Avg. $T(\mathrm{ps})$ & $k_{r}{ }^{*} 10^{8}\left(\mathrm{~s}^{-1}\right)$ & $E_{g}(\mathrm{~nm})$ & $\mu(\mathrm{D})$ & $\eta_{S R}$ \\
\hline $\mathbf{1 a}$ & Monomer & $0.025 \pm 0.002$ & $202 \pm 1$ & $1.2 \pm 0.1$ & 528 & $6.8 \pm 0.3$ & \\
\hline $\mathbf{1 a}$ & DWNT & $0.15 \pm 0.020^{\mathrm{a}}$ & $140 \pm 2$ & $20 \pm 2$ & 597 & $33 \pm 2$ & $23.2 \pm 0.8$ \\
\hline $\mathbf{1 a}$ & Bundle & $0.036 \pm 0.004$ & $81 \pm 1$ & $7.3 \pm 0.7$ & 602 & $20 \pm 1$ & $8.7 \pm 0.3$ \\
\hline $\mathbf{1 b}$ & Monomer & $0.028 \pm 0.002$ & $220 \pm 1$ & $1.3 \pm 0.1$ & 530 & $6.9 \pm 0.1$ & \\
\hline $\mathbf{1 b}$ & DWNT & $0.040 \pm 0.004^{\mathrm{a}}$ & $68 \pm 2$ & $8.1 \pm 0.7$ & 593 & $21 \pm 1$ & $9.0 \pm 0.2$ \\
\hline $\mathbf{1 b}$ & Bundle & $0.013 \pm 0.001$ & $62 \pm 1$ & $3.2 \pm 0.3$ & 603 & $13.4 \pm 0.7$ & $3.8 \pm 0.1$ \\
\hline $\mathbf{2 a}$ & Monomer & $0.33 \pm 0.025$ & $1019 \pm 3$ & $3.3 \pm 0.2$ & 625 & $14.2 \pm 0.5$ & \\
\hline $\mathbf{2 a}$ & Bundle & $0.012 \pm 0.001$ & $72 \pm 2$ & $2.6 \pm 0.3$ & 740 & $16.3 \pm 0.9$ & $1.3 \pm 0.1$ \\
\hline $\mathbf{2 b}$ & Monomer & $0.36 \pm 0.020$ & $1126 \pm 2$ & $3.2 \pm 0.2$ & 623 & $14.1 \pm 0.4$ & \\
\hline $\mathbf{2 b}$ & Bundle & $0.006 \pm 0.001$ & $70 \pm 1$ & $1.6 \pm 0.2$ & 725 & $12.2 \pm 0.6$ & $0.8 \pm 0.1$ \\
\hline
\end{tabular}

avalues taken from literature..$^{31,40}$

There is a clear correlation between the heterogeneity of self-assembly and the photophysical properties in these aggregates. For example, the bundles of the trimethine dyes deliver a lower $\eta_{S R}$ in terms of superradiance than the DWNTs. Similarly, the pentamethine bundles have a lower $\eta_{S R}$ relative to the trimethine variant, and the $\mathbf{1} \mathbf{b} / \mathbf{2} \mathbf{b}$ (brominated) bundles lower yet than 
1a/2a (chlorinated). In general, this suggests that more ordered structures yield higher superradiance. Extrapolating from this connection, the future development of molecular aggregates for photophysical applications should center around achieving uniform supramolecular structures with robust assembly. These results bolster the need for exploration in structure/property relationships between dye structures and their self-assembly, a topic that remains a fundamental challenge for this field.

\section{Conclusion}

In summary, we synthesized and aggregated two new pentamethine dyes based on the C8S3 scaffold to create two new emitters at $\sim 750 \mathrm{~nm}$. We maintain the lineshapes of the original aggregates while redshifting by $100 \mathrm{~nm}$ and found with cryoEM imaging that the pentamethine dyes mainly self-assembled into large bundles of nanotubes, as opposed to long single tubes. Photophysical characterization of these aggregates then allowed us to calculate transition dipole moments and superradiance parameters for each compound and aggregate morphology. Correlating the morphological and photophysical data, we were able conclude that the pentamethine dyes displayed different kinetics of self-assembly that resulted in generally more disordered aggregate bundles with lower superradiance relative to their trimethine comparators. Synthesizing these results, this work introduces two new NIR antennae as models for study and suggests that future works should aim for more ordered assemblies to achieve superradiant aggregates in the near-infrared.

\section{Acknowledgements}

This work was supported by NSF CHE grant no. 1905242 and a faculty research grant (UCLA Academic Senate), as well as NSF CHE-1048804. The authors acknowledge the use of instruments at the Electron Imaging Center for NanoMachines supported by the NIH (1S10RR23057) and CNSI at UCLA.

\section{References}

(1) Bricks, J. L.; Slominskii, Y. L.; Panas, I. D.; Demchenko, A. P. Fluorescent J-Aggregates of Cyanine Dyes: Basic Research and Applications Review. Methods Appl. Fluoresc. 2017, 6 (1), 012001. https://doi.org/10.1088/2050-6120/AA8D0D.

(2) Würthner, F.; Kaiser, T. E.; Saha-Möller, C. R. J-Aggregates: From Serendipitous Discovery to Supramolecular Engineering of Functional Dye Materials. Angew. Chemie Int. Ed. 2011, 50 (15), 3376-3410. https://doi.org/10.1002/ANIE.201002307.

(3) Sun, C.; Li, B.; Zhao, M.; Wang, S.; Lei, Z.; Lu, L.; Zhang, H.; Feng, L.; Dou, C.; Yin, D.; et al. J-Aggregates of Cyanine Dye for NIR-II in Vivo Dynamic Vascular Imaging beyond 1500 Nm. J. Am. Chem. Soc. 2019, 141 (49), 19221-19225. https://doi.org/10.1021/JACS.9B10043.

(4) Shakiba, M.; Ng, K. K.; Huynh, E.; Chan, H.; Charron, D. M.; Chen, J.; Muhanna, N.; Foster, F. S.; Wilson, B. C.; Zheng, G. Stable J-Aggregation Enabled Dual Photoacoustic and Fluorescence Nanoparticles for Intraoperative Cancer Imaging. Nanoscale 2016, 8 (25), 12618-12625. https://doi.org/10.1039/C5NR08165C.

(5) Saito*, K. Quenching of Excited J Aggregates on Metals by Surface Plasmon Excitations. J. Phys. Chem. B 1999, 103 (31), 6579-6583. https://doi.org/10.1021/JP991082K.

(6) Brixner, T.; Hildner, R.; Köhler, J.; Lambert, C.; Würthner, F. Exciton Transport in Molecular Aggregates - From Natural Antennas to Synthetic Chromophore Systems. Adv. Energy Mater. 2017, 7 (16), 1700236. https://doi.org/10.1002/AENM.201700236.

(7) Hans von Berlepsch, ${ }^{*}, \dagger$; Stefan Kirstein, $\ddagger$ and; Böttcher + , C. Supramolecular Structure of J-Aggregates of a Sulfonate Substituted Amphiphilic Carbocyanine Dye in Solution: Methanol-Induced Ribbon-to-Tubule Transformation. J. Phys. Chem. B 2004, 108 (48), 18725-18733. https://doi.org/10.1021/JP046546F.

(8) Deshmukh, A. P.; Koppel, D.; Chuang, C.; Cadena, D. M.; Cao, J.; Caram, J. R. Design Principles for Two-Dimensional Molecular Aggregates Using Kasha's Model: Tunable Photophysics in Near and Short-Wave Infrared. 2019. https://doi.org/10.1021/acs.jpcc.9b05060.

Engel, G. S.; Calhoun, T. R.; Read, E. L.; Ahn, T. K.; Mančal, T.; Cheng, Y. C.; Blankenship, R. E.; Fleming, G. R. Evidence for Wavelike Energy Transfer through Quantum Coherence in Photosynthetic Systems. Nature 2007, 446 (7137), 782-786. https://doi.org/10.1038/nature05678.

(10) Scholes, G. D.; Fleming, G. R.; Olaya-Castro, A.; Van Grondelle, R. Lessons from Nature about Solar Light Harvesting. Nature Chemistry. Nature Publishing Group October 23, 2011, pp 763-774. https://doi.org/10.1038/nchem.1145.

(11) Freyria, F. S.; Cordero, J. M.; Caram, J. R.; Doria, S.; Dodin, A.; Chen, Y.; Willard, A. P.; Bawendi, M. G. Near-Infrared Quantum Dot Emission Enhanced by Stabilized Self-Assembled J-Aggregate Antennas. Nano Lett. 2017, 17 (12), 7665- 
7674. https://doi.org/10.1021/ACS.NANOLETT.7B03735.

(12) Caram, J. R.; Engel, G. S. Extracting Dynamics of Excitonic Coherences in Congested Spectra of Photosynthetic Light Harvesting Antenna Complexes. Faraday Discuss. 2011, 153 (0), 93-104. https://doi.org/10.1039/C1FD00049G.

(13) Wang, C.; Weiss, E. A. Accelerating FRET between Near-Infrared Emitting Quantum Dots Using a Molecular J-Aggregate as an Exciton Bridge. Nano Lett. 2017, 17 (9), 5666-5671. https://doi.org/10.1021/ACS.NANOLETT.7B02559.

(14) Doria, S.; Sinclair, T. S.; Klein, N. D.; Bennett, D. I. G.; Chuang, C.; Freyria, F. S.; Steiner, C. P.; Foggi, P.; Nelson, K. A.; Cao, J.; et al. Photochemical Control of Exciton Superradiance in Light-Harvesting Nanotubes. ACS Nano 2018, 12 (5), 4556-4564. https://doi.org/10.1021/acsnano.8b00911.

(15) Chuang, C.; Lee, C. K.; Moix, J. M.; Knoester, J.; Cao, J. Quantum Diffusion on Molecular Tubes: Universal Scaling of the 1D to 2D Transition. 2016. https://doi.org/10.1103/PhysRevLett.116.196803.

(16) Berlepsch, H. v.; Böttcher, C. Tubular J-Aggregates of a New Thiacarbocyanine Cy5 Dye for the Far-Red Spectral Region - a Spectroscopic and Cryo-Transmission Electron Microscopy Study. Phys. Chem. Chem. Phys. 2018, 20 (28), 1896918977. https://doi.org/10.1039/C8CP03378A.

(17) Chen, Z.; Liu, Y.; Wagner, W.; Stepanenko, V.; Ren, X.; Ogi, S.; Würthner, F. Near-IR Absorbing J-Aggregate of an Amphiphilic BF2-Azadipyrromethene Dye by Kinetic Cooperative Self-Assembly. Angew. Chemie Int. Ed. 2017, 56 (21), 5729-5733. https://doi.org/10.1002/ANIE.201701788.

(18) Wenus, J.; Ceccarelli, S.; Lidzey, D. G.; Tolmachev, A. I.; Slominskii, J. L.; Bricks, J. L. Optical Strong Coupling in Microcavities Containing J-Aggregates Absorbing in near-Infrared Spectral Range. Org. Electron. 2007, 8 (2-3), $120-126$. https://doi.org/10.1016/J.ORGEL.2006.06.006.

(19) Hilderbrand, S. A.; Weissleder, R. Near-Infrared Fluorescence: Application to in Vivo Molecular Imaging. Curr. Opin. Chem. Biol. 2010, 14 (1), 71-79. https://doi.org/10.1016/J.CBPA.2009.09.029.

(20) Lim, Y. T.; Kim, S.; Nakayama, A.; Stott, N. E.; Bawendi, M. G.; Frangioni, J. V. Selection of Quantum Dot Wavelengths for Biomedical Assays and Imaging: Mol. Imaging 2003, 2 (1), 50-64. https://doi.org/10.1162/15353500200302163.

(21) Rondão, R.; Frias, A. R.; Correia, S. F. H.; Fu, L.; Bermudez, V. de Z.; André, P. S.; Ferreira, R. A. S.; Carlos, L. D. HighPerformance Near-Infrared Luminescent Solar Concentrators. ACS Appl. Mater. Interfaces 2017, 9 (14), $12540-12546$. https://doi.org/10.1021/ACSAMI.7B02700.

(22) Pawlik, A.; Kirstein, S.; De Rossi, U.; Daehne, S. Structural Conditions for Spontaneous Generation of Optical Activity in JAggregates. J. Phys. Chem. B 1997, 101, 5646-5651.

(23) Eisele, D. M.; Cone, C. W.; Bloemsma, E. A.; Vlaming, S. M.; Van Der Kwaak, C. G. F.; Silbey, R. J.; Bawendi, M. G.; Knoester, J.; Rabe, J. P.; Vanden Bout, D. A. Utilizing Redox-Chemistry to Elucidate the Nature of Exciton Transitions in Supramolecular Dye Nanotubes. Nat. Chem. 2012, 4 (8), 655-662. https://doi.org/10.1038/nchem.1380.

(28) Caram, J. R.; Doria, S.; Eisele, D. M.; Freyria, F. S.; Sinclair, T. S.; Rebentrost, P.; Lloyd, S.; Bawendi, M. G. RoomTemperature Micron-Scale Exciton Migration in a Stabilized Emissive Molecular Aggregate. Nano Lett. 2016, 16 (11), 6808-6815. https://doi.org/10.1021/acs.nanolett.6b02529.

(29) Yuen-Zhou, J.; Arias, D. H.; Eisele, D. M.; Steiner, C. P.; Krich, J. J.; Bawendi, M. G.; Nelson, K. A.; Aspuru-Guzik, A. Coherent Exciton Dynamics in Supramolecular Light-Harvesting Nanotubes Revealed by Ultrafast Quantum Process 
Tomography. ACS Nano 2014, 8 (6), 5527-5534. https://doi.org/10.1021/NN406107Q.

(30) Kriete, B.; Lüttig, J.; Kunsel, T.; Malý, P.; Jansen, T. L. C.; Knoester, J.; Brixner, T.; Pshenichnikov, M. S. Interplay between Structural Hierarchy and Exciton Diffusion in Artificial Light Harvesting. https://doi.org/10.1038/s41467-019-12345-9.

(31) Rn Kriete, B.; Bondarenko, A. S.; Jumde, V. R.; Franken, L. E.; Minnaard, A. J.; Jansen, T. L. C.; Knoester, J.; Pshenichnikov, M. S. Steering Self-Assembly of Amphiphilic Molecular Nanostructures via Halogen Exchange. J. Phys. Chem. Lett 2017. https://doi.org/10.1021/acs.jpclett.7b00967.

(32) Roth, S. M.; Press, D. J.; Heyne, B.; Sutherland, T. C. Synthetic Access to Benzimidacarbocyanine Dyes to Tailor Their Aggregation Properties. J. Org. Chem. 2021, 86, 8651. https://doi.org/10.1021/ACS.JOC.1C00385.

(33) Bricks, J. L.; Kachkovskii, A. D.; Slominskii, Y. L.; Gerasov, A. O.; Popov, S. V. Molecular Design of near Infrared Polymethine Dyes: A Review. Dyes and Pigments. 2015. https://doi.org/10.1016/j.dyepig.2015.05.016.

(34) Clark, K. A.; Cone, C. W.; Bout, D. A. Vanden. Quantifying the Polarization of Exciton Transitions in Double-Walled Nanotubular J-Aggregates. J. Phys. Chem. C 2013, 117 (50), 26473-26481. https://doi.org/10.1021/jp409573h.

Deshmukh, A. P.; Bailey, A. D.; Forte, L. S.; Shen, X.; Geue, N.; Sletten, E. M.; Caram, J. R. Thermodynamic Control over Molecular Aggregate Assembly Enables Tunable Excitonic Properties across the Visible and Near-Infrared. J. Phys. Chem. Lett. 2020, 11 (19), 8026-8033. https://doi.org/10.1021/acs.jpclett.0c02204.

(36) Akins, D. L. Superradiance of Aggregated Thiacarbocyanine Molecules. 1999. https://doi.org/10.1021/jp991627a.

(37) Zhao, H.; Zhao, Y.; Song, Y.; Zhou, M.; Lv, W.; Tao, L.; Feng, Y.; Song, B.; Ma, Y.; Zhang, J.; et al. Strong Optical Response and Light Emission from a Monolayer Molecular Crystal. Nat. Commun. 2019101 2019, 10 (1), 1-9. https://doi.org/10.1038/s41467-019-13581-9.

(38) Friedman, H.; Cosco, E.; Atallah, T.; Jia, S.; Sletten, E.; Caram, J. Establishing Design Principles for Emissive Organic SWIR Chromophores from Energy Gap Laws. Chem 2021, 7, 1-18. https://doi.org/10.26434/CHEMRXIV.14374493.V1.

(39) Tsurumi, T.; Hirayama, H.; Vacha, M.; Taniyama, T. Nanoscale Physics for Materials Science; Taylor \& Francis: Abingdon, 2010.

(40) Freyria, F. S.; Cordero, J. M.; Caram, J. R.; Doria, S.; Dodin, A.; Chen, Y.; Willard, A. P.; Bawendi, M. G. Near-Infrared Quantum Dot Emission Enhanced by Stabilized Self-Assembled J-Aggregate Antennas. Nano Lett. 2017, 17 (12), 7665 7674. https://doi.org/10.1021/ACS.NANOLETT.7B03735. 